Website: http://revistas.lamolina.edu.pe/index.php/acu/index

(C) Universidad Nacional Agraria La Molina, Lima - Perú

\title{
Efectos del cadmio sobre el crecimiento y la composición elemental de la alfalfa en cultivo de arena
}

\author{
Effects of cadmium on the growth and elemental composition of alfalfa in sand culture
}

\author{
Klaus P. Raven Willwater ${ }^{1}$
}

\begin{abstract}
Resumen
El cadmio $(\mathrm{Cd})$ es un elemento traza natural que puede provocar toxicidad en las plantas. Los objetivos de esta investigación fueron evaluar los efectos del nivel de $\mathrm{Cd}$ en un medio de arena sobre el crecimiento de la alfalfa y su contenido de Cd, calcio $(\mathrm{Ca})$, cobre $(\mathrm{Cu})$, hierro $(\mathrm{Fe})$, potasio $(\mathrm{K})$, magnesio $(\mathrm{Mg})$, manganeso $(\mathrm{Mn})$, nitrógeno $(\mathrm{N})$, sodio $(\mathrm{Na})$, fósforo (P), azufre (S) y zinc (Zn). Los tratamientos fueron 3 dosis de Cd: 0, 5 y $25 \mathrm{mg} \mathrm{kg}^{-1}$ arena. Semillas de alfalfa fueron sembradas en estos medios, en base a un diseño de bloques completos randomizados con tres repeticiones. Las plantas se dejaron crecer por 60 días, siendo periódicamente regadas y fertilizadas. A la cosecha, se evaluaron el contenido de clorofila, la materia seca de raíces y parte aérea y las concentraciones de los mencionados elementos en la materia seca. A mayor dosis de Cd se observaron notorias reducciones del contenido de clorofila y del crecimiento vegetal. Ninguna de las plantas, ya sea expuestas o no a este elemento, mostraron carencia o exceso de algún nutriente mineral estudiado. La adición de Cd tendió a provocar la concentración de elementos nutritivos en la reducida materia seca y la acumulación preferencial de estos en la parte aérea a expensas de las raíces. No se observó ningún efecto específico notorio de este elemento sobre los nutrientes minerales evaluados. Aparentemente, una limitada fotosíntesis en las plantas fue el daño directo principal del $\mathrm{Cd}$ en este estudio.
\end{abstract}

Palabras clave: cadmio; alfalfa; crecimiento; elemento; nutriente; arena.

\begin{abstract}
Cadmium $(\mathrm{Cd})$ is a natural trace element that may cause toxicity in plants. The objectives of this research were to evaluate the effects of the $\mathrm{Cd}$ level in a sand medium on the growth of alfalfa and its content of Cd, calcium (Ca), copper $(\mathrm{Cu})$, iron $(\mathrm{Fe})$, potassium $(\mathrm{K})$, magnesium $(\mathrm{Mg})$, manganese $(\mathrm{Mn})$, nitrogen $(\mathrm{N})$, sodium $(\mathrm{Na})$, phosphorus $(\mathrm{P})$, sulfur $(\mathrm{S})$ y zinc $(\mathrm{Zn})$. The treatments were $3 \mathrm{Cd}$ doses: 0,5 y $25 \mathrm{mg} \mathrm{kg}^{-1}$ sand. Alfalfa seeds were sown in these media, according to a randomized complete block design with 3 replicates. The plants were allowed to grow for 60 days, being periodically watered and fertilized. At harvest, the chlorophyll content, the root and shoot dry matter, and the concentrations of the mentioned elements in dry matter were evaluated. Notorious decreases in chlorophyll content and plant growth were observed at higher $\mathrm{Cd}$ doses. None of the plants, either exposed or not to $\mathrm{Cd}$, showed shortage or excess of any of the studied nutrient elements. The addition of $\mathrm{Cd}$ tended to concentrate the nutrient elements in the reduced dry matter and to preferentially accumulate them in the shoots at the expense of roots. No specific notorious effect of this element was observed on the evaluated mineral nutrients. Apparently, limited photosynthesis in plants was the main direct damage of $\mathrm{Cd}$ in this study.
\end{abstract}

Keywords: cadmium; alfalfa; growth; element; nutrient; sand.

\section{Introducción}

El cadmio (Cd) es un elemento traza en el ambiente natural, incluyendo suelos y plantas (Kabata-Pendias \& Szteke, 2015). Este elemento no es esencial para la vida, pero ha adquirido interés debido al efecto perjudicial que causa en animales y plantas cuando se almacena en sus tejidos.

La acumulación de $\mathrm{Cd}$ en las plantas tiende a alterar su metabolismo y fisiología y, eventualmente, puede causar su muerte (DalCorso et al., 2013; Irfan et al., 2013). Sin embargo, una cantidad significativa de este debe ser incorporada para que se produzcan daños notorios en su crecimiento y desarrollo (Bingham et al., 1975).

Un contenido elevado de $\mathrm{Cd}$ en especies vegetales afecta su composición elemental (DalCorso et al., 2013; Irfan et al., 2013). La mayor parte de la información existente sobre este tema se originó de datos complementarios generados en estudios acerca de algún aspecto de la toxicidad del $\mathrm{Cd}$ en plantas. Estos trabajos generalmente presentaron resultados acerca de un número restringido de elementos nutritivos. Investigaciones específicamente diseñadas para estudiar el efecto del Cd sobre la nutrición mineral de las plantas y que incluyeron a seis o mas elementos nutritivos han sido llevados a cabo en solución nutritiva con alfalfa (Ibekwe et al., 1996), Pfaffia glomerata (Gomes et al., 2013) y tomate (Hédiji et al., 2015). Estudios de estas características no se han realizado en suelos o sustratos relacionados. Por otra parte, los trabajos hidropónicos mencionados han producido información limitada acerca 
del nitrógeno $(\mathrm{N})$, sodio $(\mathrm{Na})$, fósforo $(\mathrm{P})$ y azufre $(\mathrm{S})$. Finalmente, los efectos provocados por el $\mathrm{Cd}$ no siempre han tenido el mismo patrón de respuesta para cada elemento nutritivo en las plantas.

El conocimiento del efecto del Cd sobre la composición elemental de las plantas es útil porque estas son utilizadas para el consumo humano y del ganado. Además, las plantas son empleadas en algunas tecnologías de remediación de suelos contaminados. Esta información permite plantear estrategias para mejorar el contenido de nutrientes y reducir la acumulación de $\mathrm{Cd}$ en el producto cosechado y para mejorar la supervivencia de especies vegetales en suelos contaminados. La alfalfa es comúnmente utilizada para alimentar animales domésticos. Esta especie vegetal también ha sido recomendada para fitoestabilizar metales pesados en suelos contaminados (Motesharezadeh et al., 2010).

Los objetivos del presente trabajo fueron evaluar los efectos del nivel de $\mathrm{Cd}$ en un sustrato de arena sobre el crecimiento de la alfalfa y su contenido de $\mathrm{Cd}$, calcio $(\mathrm{Ca})$, cobre $(\mathrm{Cu})$, hierro $(\mathrm{Fe})$, potasio $(\mathrm{K})$, magnesio $(\mathrm{Mg})$, manganeso $(\mathrm{Mn}), \mathrm{N}, \mathrm{Na}, \mathrm{P}, \mathrm{S}$ y zinc $(\mathrm{Zn})$.

\section{Materiales y métodos}

Para lograr el objetivo de la presente investigación, se ejecutó un experimento con 3 tratamietres y 3 repeticiones. Las 9 unidades experimentales fueron distribuidas en un patio experimental en Lima, Perú, en base a un diseño de bloques completos randomizados. Los tratamientos fueron 3 dosis de aplicación de Cd: 0,5 y $25 \mathrm{mg} \mathrm{kg}^{-1}$ sustrato. Estas dosis representaron las situaciones prácticas de suelos con grados de contaminación bajo, moderado y alto, respectivamente. Cada unidad experimental consistió de una maceta conteniendo 20 plantas de alfalfa creciendo en $1 \mathrm{~kg}$ de arena tratada.

La arena utilizada presentó un $\mathrm{pH}$ igual a 8,22, una conductividad eléctrica en extracto de saturación igual $1,31 \mathrm{dS} \mathrm{m}^{-1}$ y un contenido de carbonatos igual a $31 \mathrm{~g} \mathrm{~kg}^{-1}$. Estos parámetros fueron determinados, así como descrito por Bazán (1996). Nueve porciones de $1 \mathrm{~kg}$ de arena seca al aire fueron separadas y colocadas en bolsas plásticas. A cada porción, se adicionaron $25 \mathrm{~mL}$ de solución de $\mathrm{CdCl}_{2} \cdot 2,5 \mathrm{H}_{2} \mathrm{O}$ para lograr la dosis de Cd correspondiente. Después de homogeneizar cada porción de arena tratada, estas fueron transferidas a recipientes de plástico de $1 \mathrm{~L}$ de capacidad y llevadas a capacidad de campo.

Al cabo de 2 días de equilibración, se inició el experimento con la siembra de 40 semillas de alfalfa de la variedad San Pedro en cada recipiente. A los 26 días después de la siembra, se redujo el número de plantas de alfalfa a 20 por maceta. Durante el ensayo, cada unidad experimental fue regada periódicamente con agua desionizada para evitar estrés hídrico. Además, todas las macetas recibieron un suplemento de 150,40,100, 50, 20, 27, 20, 10, 4 y 1,2 $\mathrm{mg} \mathrm{maceta}^{-1}$ de N, P, K, Ca, Mg, S, Fe, Mn, Zn y Cu, respectivamente. Durante el bioensayo, la temperatura y humedad relativa medias en el patio experimental fueron $25,9^{\circ} \mathrm{C}$ y $64,5 \%$, respectivamente.
Un día antes de la cosecha, se evaluó el contenido de clorofila en las plantas de alfalfa. Para ello, se realizaron 10 determinaciones por maceta, siendo cada una hecha en un tallo seleccionado al azar. Cada determinación se efectuó en el foliolo central de la hoja localizada en la parte media del tallo. Las mediciones se ejecutaron con un medidor SPAD 502 (Spectrum Technologies, Inc.). Los resultados fueron reportados como promedios de 10 determinaciones por maceta y expresados en unidades SPAD.

La cosecha se realizó a los 60 días después de la siembra. Esta se inició con la colección de biomasa de la parte aérea, mediante corte a nivel de la corona de la planta. Por otra parte, la biomasa de las raíces se obtuvo en base al siguiente procedimiento: (i) desprendimiento de raíces de la arena después de sumergir la maceta íntegra en un balde con agua potable, (ii) colección del material radicular desprendido mediante paso del contenido del balde a través de un tamiz de plástico, y (iii) lavado de raíces colectadas sobre el tamiz bajo chorro de agua potable. La biomasa separada de parte aérea y raíces fue lavada con agua desionizada y colocada en bolsas de papel.

Las bolsas de papel conteniendo la biomasa cosechada fueron colocadas en una estufa graduada a $68^{\circ} \mathrm{C}$ y sometidas a secado hasta obtener peso constante en el tiempo. Los pesos de la materia seca así obtenida se registraron. Estos resultados se utilizaron para calcular la materia seca total y el cociente de materia seca de parte aérea a raíces para cada unidad experimental.

Las muestras de materia seca fueron molidas, usando un mini molino Wiley (Thomas Scientific) con malla de 40 mesh. La materia seca molida fue colectada en bolsas de polietileno. Luego, esta fue empleada para determinar sus concentraciones de $\mathrm{Cd}, \mathrm{Ca}, \mathrm{Cu}, \mathrm{Fe}, \mathrm{K}, \mathrm{Mg}, \mathrm{Mn}, \mathrm{N}, \mathrm{Na}, \mathrm{P}, \mathrm{S}$ y $\mathrm{Zn}$. Con los datos de concentración para cada elemento químico en la materia seca de raíces y parte aérea, se procedió a calcular las extracciones de este en raíces, parte aérea y total de planta y el cociente de extracciones de parte aérea a raíces.

La concentración de $\mathrm{N}$ en la materia seca se determinó mediante el método de micro-Kjeldahl. Cada muestra de $0,1 \mathrm{~g}$ fue digestada con $\mathrm{H}_{2} \mathrm{SO}_{4}$ en presencia de una mezcla catalizadora de $\mathrm{CuSO}_{4}: \mathrm{K}_{2} \mathrm{SO}_{4}$ 1:9 en un tubo de digestión de $100 \mathrm{ml}$ colocado en un equipo Turbotherm (Gerhardt Analytical Systems). Al cabo de 1,5 horas, se destiló la muestra digestada en un equipo Vapodest 20s (Gerhardt Analytical Systems), y el $\mathrm{NH}_{3}$ desprendido fue colectado en $\mathrm{H}_{3} \mathrm{BO}_{3}$ al $2 \%$ conteniendo los indicadores rojo de metilo y azul de metileno. El $\mathrm{NH}_{3}$ colectado fue titulado con $\mathrm{H}_{2} \mathrm{SO}_{4} 0,02 \mathrm{~N}$.

Las concentraciones de los demás elementos químicos fueron determinadas a partir de extractos de digestión húmeda de la materia seca. La digestión húmeda se realizó usando $\mathrm{HNO}_{3}$ y $\mathrm{HClO}_{4}$ de acuerdo con un procedimiento modificado de Jones y Case (1990). Las modificaciones fueron: (i) tamaño de muestra de $1 \mathrm{~g}$, (ii) uso de $10 \mathrm{~mL}$ de una mezcla 5:1 de $\mathrm{HNO}_{3}: \mathrm{HClO}_{4}$ concentrados desde el inicio y sin adiciones posteriores de ácidos, y (iii) filtrado y dilución final a $25 \mathrm{~mL}$. Las concentraciones de $\mathrm{Cd}, \mathrm{Ca}, \mathrm{Cu}$, 
Fe, K, Mg, Mn y Zn en los extractos fueron determinadas por espectrofotometría de absorción atómica en llama de acetileno-aire, utilizando las líneas de absorción de $228,8,422,7,324,8,248,3,766,5,285,2,279,5$ у 213,9 $\mathrm{nm}$, respectivamente. Estos análisis se efectuaron con dos instrumentos: Perkin Elmer 3100 AAS y Perkin Elmer AAnalyst 200 AAS. Para $\mathrm{Ca}$ y $\mathrm{Mg}$, las lecturas fueron hechas en extractos diluidos que contenían $1000 \mathrm{mg}$ $\mathrm{L}^{-1}$ de lantano (La). Aparte de estas consideraciones, la operación de estos instrumentos se realizó de acuerdo a las recomendaciones generales del fabricante. Por otra parte, la concentración de $\mathrm{P}$ en los extractos se evaluó mediante espectrofotometría de absorción molecular UV/visible del complejo molibdofosfórico, así como descrito por Bazán (1996). Finalmente, la concentración de $\mathrm{S}$ en los extractos se determinó mediante turbidimetría, así como detallado por Bazán (1996).

La evaluación estadística del experimento se realizó mediante el análisis de variancias y la prueba de comparación múltiple de Tukey para cada parámetro determinado o calculado. Para ello, se siguieron los lineamientos descritos por Ott y Longnecker (2016). En los casos especiales de parámetros con alto coeficiente de variabilidad y falta de significación estadística con los procedimientos paramétricos, el análisis estadístico se complementó con la prueba de Friedman, así como descrito por Hollander et al. (2014).

\section{Resultados y discusión}

El incremento del nivel de $\mathrm{Cd}$ en la arena tuvo un efecto depresivo sobre el crecimiento de las plantas de alfalfa, como mostrado en la Tabla 1 . Al agregar $5 \mathrm{mg} \mathrm{Cd} \mathrm{kg}{ }^{-1}$ arena, las producciónes de materia seca de raíces, parte aérea y total se redujeron a 82,87 y $85 \%$, respectivamente, de los valores de plantas testigo. Al aplicar $25 \mathrm{mg} \mathrm{Cd}$ $\mathrm{kg}^{-1}$ arena, estas llegaron a disminuir hasta 60, 71 y 67 $\%$, respectivamente, con respecto a plantas no tratadas con Cd. Estas reducciones tan notorias, no lograron significación estadística en análisis de variancias y pruebas de Tukey, excepto para la parte aérea. Sin embargo, la prueba no paramétrica de Friedman arrojó diferencias significativas entre los tratamientos para las tres variables discutidas. El efecto negativo de la aplicación de $\mathrm{Cd}$ sobre el crecimiento de las plantas ha sido comúnmente documentado en alfalfa (Dražić et al., 2006; Ghnaya et al., 2015; Ibekwe et al., 1996; Motesharezadeh et al., 2010) y otras especies vegetales (Ahmad et al., 2016; Arshad et al., 2016; Astolfi et al., 2014; Borišev et al., 2016; Dias et al., 2013; Eller y Brix, 2016; Gill et al., 2012; Gomes et al., 2013; Li et al., 2016; Pérez-Romero et al., 2016; Sebastian y Prasad, 2015; Yang et al., 2016; Zhang et al., 2014). Por otra parte, el cociente de materia seca de parte aérea a raíces aumentó en un $18 \%$ al aplicar la dosis mas alta de $\mathrm{Cd}$ y no alcanzó significación estadística. Ningún resultado comparable pudo encontrarse en otros trabajos con alfalfa. Sin embargo, reportes de disminución, aunque no siempre significativos, de este parámetro ante la adición de Cd existen para especies vegetales distintas (Gomes et al., 2013; Yang et al., 2016).

La aplicación de $25 \mathrm{mg} \mathrm{Cd} \mathrm{kg}^{-1}$ arena disminuyó el contenido de clorofila en los foliolos de las plantas de alfalfa hasta un $85 \%$ del valor de plantas sometidas a menores dosis, como mostrado en la Tabla 1. Esta reducción alcanzó significación estadística. Resultados similares han sido comúnmente observados en alfalfa (Ibekwe et al., 1996) y otras especies vegetales (Ahmad et al., 2016; Arshad et al., 2016; Astolfi et al., 2014; Borišev et al., 2016; Dias et al., 2013; Gill et al., 2012; Li et al., 2016; Pérez-Romero et al., 2016; Sebastian y Prasad, 2015; Zhang et al., 2014). Una fotosíntesis restringida generalmente acompañó al menor contenido de clorofila (Arshad et al., 2016; Dias et al., 2013; Gill et al., 2012; Pérez-Romero et al., 2016). Esto es coherente con la reducida materia seca de las plantas de alfalfa de este estudio.

La aplicación de $\mathrm{Cd}$ afectó de manera notoria y estadísticamente significativa a todos los parámetros de concentración y extracción evaluados para este elemento, como ilustrado en la Tabla 1. Plantas de alfalfa no tratadas tuvieron concentraciones de $\mathrm{Cd}$ parecidas en la materia seca de raíces y parte aérea. Estos valores aumentaron 480 y 16 veces, respectivamente, al suministrar la dosis mas alta de Cd. Resultados similares se han reportado frecuentemente para alfalfa (Dražić et al., 2006; Ghnaya et al., 2015; Ibekwe et al., 1996; Motesharezadeh et al., 2010) y otras especies de plantas (Ahmad et al., 2016; Arshad et al., 2016; Astolfi et al., 2014; Borišev et al., 2016; Chaoui et al., 1997; Dias et al., 2013; Gill et al., 2012; Gomes et al., 2013; Lopes Júnior et al., 2014; PérezRomero et al., 2016; Sebastian y Prasad, 2015; Yang et al., 2016). En estas investigaciones, también se observó que la concentración de $\mathrm{Cd}$ en las raíces tendía a ser mucho mayor que en la parte aérea.

La extracción total de Cd de las plantas de alfalfa se incrementó a unas 124 veces el valor del testigo al aplicar la dosis mayor de Cd. Esta extracción alcanzó un 12 y $6 \%$ del Cd aplicado en las macetas tratadas con 5 y 25

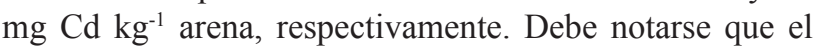
$\mathrm{Cd}$ suministrado tuvo una disponibilidad relativamente alta para las plantas, porque fue aplicado como sal muy soluble en agua y el periodo de equilibración y adsorción del mismo con la arena, antes de iniciar el experimento, fue intencionalmente corto. Por ello, los citados porcentajes deben interpretarse como altos. Además, al aumentar la dosis de $\mathrm{Cd}$ en la arena, el cociente de extracción parte aérea a raíces para este elemento se redujo notoriamente. En plantas no tratadas, la extracción de $\mathrm{Cd}$ en la parte aérea fue aproximadamente $50 \%$ mayor que en las raíces. Sin embargo, al aumentar la dosis de Cd, este se acumuló preferentemente en las raíces, cuyo contenido alcanzó un $95 \%$ del total extraído. Tendencias variadas han sido observadas en estudios con alfalfa (Ghnaya et al., 2015; 
Tabla 1. Análisis de variancias y promedios para parámetros de crecimiento y de contenido de Cd en plantas de alfalfa

\begin{tabular}{|c|c|c|c|c|c|c|c|}
\hline \multirow{2}{*}{ Parámetro } & \multicolumn{3}{|c|}{ Cuadrados Medios } & \multirow{2}{*}{$\begin{array}{c}\text { C. V. }{ }^{1} \\
(\%)\end{array}$} & \multicolumn{3}{|c|}{ Promedios para Dosis Cd } \\
\hline & Bloques & Dosis Cd & Error Exp. & & $0 \mathrm{mg} \mathrm{kg}^{-1}$ & $5 \mathrm{mg} \mathrm{kg}^{-1}$ & $25 \mathrm{mg} \mathrm{kg}^{-1}$ \\
\hline Materia Seca Raíces (g maceta ${ }^{-1}$ ) & $0,552 \mathrm{NS}, 2$ & $1,370^{\mathrm{NS} 2}$ & 0,342 & 21,62 & $3,36 \mathrm{a}^{3}$ & $2,74 \mathrm{a}$ & $2,01 \mathrm{a}$ \\
\hline Materia Seca Parte Aérea $\left(\mathrm{g} \mathrm{maceta}^{-1}\right)$ & $0,837^{\mathrm{NS}}$ & $2,338^{\mathrm{NS}}$ & 0,344 & 11,13 & $6,13 \mathrm{a}$ & $5,32 \mathrm{ab}$ & $4,36 b$ \\
\hline Materia Seca Total $\left(\right.$ g maceta $\left.^{-1}\right)$ & 2,47 NS & $7,29 \mathrm{Ns}$ & 1,22 & 13,82 & $9,49 \mathrm{a}$ & $8,06 \mathrm{a}$ & $6,38 \mathrm{a}$ \\
\hline Materia Seca Parte Aérea/Raíces & $0,0605^{\mathrm{NS}}$ & $0,0802 \mathrm{NS}$ & 0,0853 & 14,54 & $1,84 \mathrm{a}$ & $2,01 \mathrm{a}$ & $2,17 \mathrm{a}$ \\
\hline Clorofila (unidades SPAD) & $4,78^{\mathrm{NS}}$ & $34,99 *$ & 1,22 & 2,95 & $38,9 \mathrm{a}$ & $40,0 \mathrm{a}$ & $33,6 b$ \\
\hline Concentración Cd Materia Seca Raíces (mg kg-1) & 2231 NS & $416626^{*}$ & 5234 & 23,20 & $1,51 b$ & $208,83 b$ & $725,16 a$ \\
\hline Concentración Cd Materia Seca Parte Aérea $\left(\mathrm{mg} \mathrm{kg}^{-1}\right)$ & $1,14 \mathrm{Ns}$ & $251,77 *$ & 1,09 & 11,37 & $1,19 \mathrm{c}$ & $7,17 b$ & $19,17 \mathrm{a}$ \\
\hline Extracción Cd Total ( $\mu$ g maceta $\left.{ }^{-1}\right)$ & $1258^{\mathrm{NS}}$ & $1748678 *$ & 374 & 2,73 & $12,3 \mathrm{c}$ & $587,6 b$ & $1524,9 \mathrm{a}$ \\
\hline Extracción Cd Parte Aérea/Raíces & 0,0462 Ns & $1,9757 *$ & 0,0454 & 40,05 & $1,4690 \mathrm{a}$ & $0,0692 b$ & $0,0576 \mathrm{~b}$ \\
\hline
\end{tabular}

1 Coeficiente de variabilidad.

2 Prueba de F para $\alpha$ igual a 0,05 : no significativa (NS) o significativa $\left(^{*}\right)$.

3 Prueba de Tukey entre promedios para $\alpha$ igual a 0,05 : letras distintas indican grupos de tratamientos significativamente diferentes.

Motesharezadeh et al., 2010). Los resultados de esta y otras investigaciones (Elouear et al., 2016; Motesharezadeh et al., 2010) sugieren que el paso del Cd de raíces a forraje es significativamente restringido en la alfalfa. Además, esta planta sería útil para fitoestabilizar, mas que fitoextraer, el $\mathrm{Cd}$ en suelos contaminados.

Plantas de alfalfa severamente intoxicadas con $\mathrm{Cd}$ han mostrado detención del crecimiento, clorosis severa generalizada, hojas maduras con necrosis internerval y hojas jóvenes cloróticas y deformadas (Ibekwe et al., 1996). En el presente estudio, las plantas tratadas con $25 \mathrm{mg} \mathrm{Cd}$ $\mathrm{kg}^{-1}$ arena no llegaron a mostrar estos síntomas. Además, la concentración de $\mathrm{Cd}$ en la materia seca de la parte aérea no alcanzó el nivel crítico de $50 \mathrm{mg} \mathrm{kg}^{-1}$ reportado por Ibekwe et al. (1996). Finalmente, la producción total de materia seca tampoco disminuyó hasta un $25 \%$ del valor del testigo, como observado en el citado estudio.

La influencia de la adición de $\mathrm{Cd}$ al sustrato sobre el contenido de $\mathrm{Ca}$ en las plantas de alfalfa se muestra en la Tabla 2. La concentración de Ca en la materia seca de raíces no fue afectada significativamente por la dosis de Cd aplicado. Sin embargo, la aplicación de Cd aumentó en mas de $25 \%$ la concentración de Ca en la materia seca de la parte aérea, lo cual logró ser estadísticamente relevante. En estudios previos con alfalfa, se observó que una mayor dosis de Cd causó una reducción de la concentración de Ca en raíces (Dražić et al., 2006) y un aumento (Dražić et al., 2006; Ibekwe et al., 1996) o disminución (Ghnaya et al., 2015) de aquella en la parte aérea. Reportes en otras especies vegetales, muestran efectos variables de la adición de Cd sobre la concentración de Ca en raíces y parte aérea (Ahmad et al., 2016; Arshad et al., 2016; Chaoui et al., 1997; Dias et al., 2013; Eller y Brix, 2016; Gomes et al., 2013; Hédiji et al., 2015; Li et al., 2016; Pérez-Romero et al., 2016).

La variación del contenido de $\mathrm{Cu}$ en plantas de alfalfa ante la aplicación de Cd se presenta en la Tabla 2. La concentración de $\mathrm{Cu}$ en la materia seca de raíces aumentó gradualmente al adicionar mas $\mathrm{Cd}$ al sustrato, llegando a ser hasta $58 \%$ mayor que en plantas testigo. Este incremento no alcanzó significación con las pruebas estadísticas empleadas. Una tendencia similar fue reportada en alfalfa
(Motesharezadeh et al., 2010) y otras especies vegetales (Chaoui et al., 1997; Gomes et al., 2013; Lopes Júnior et al., 2014; Hédiji et al., 2015). Sin embargo, también se ha observado el patrón opuesto (Astolfi et al., 2014; Wu et al., 2003). En contraste, la adición de Cd redujo ligeramente, sin significación estadística, la concentración de $\mathrm{Cu}$ en la materia seca de la parte aérea. Resultados similares han sido reportados en otros estudios con alfalfa (Ibekwe et al., 1996; Motesharezadeh et al., 2010). En otras especies vegetales, se ha observado que la adición de $\mathrm{Cd}$ causó una disminución o ningún efecto sobre el nivel de $\mathrm{Cu}$ en la parte aérea (Astolfi et al., 2014; Chaoui et al., 1997; Gomes et al., 2013; Hédiji et al., 2015; Lopes Júnior et al., 2014; Wu et al., 2003).

Los datos de concentración de Fe en las raíces de las plantas de alfalfa tuvieron que ser excluidos del análisis por mostrar muy alta variabilidad y contaminación. El efecto de la adición de Cd sobre el contenido de Fe en la parte aérea de las plantas de alfalfa se muestra en la Tabla 2. La dosis de aplicación de Cd no impactó de manera marcada ni estadísticamente significativa la concentración de Fe en la materia seca de la parte aérea. En otros estudios con alfalfa (Dražić et al., 2006; Ghnaya et al., 2015; Ibekwe et al., 1996; Motesharezadeh et al., 2010), se han reportado observaciones contradictorias. Sin embargo, experimentos con otras plantas (Arshad et al., 2016; Astolfi et al., 2014; Chaoui et al., 1997; Dias et al., 2013; Gomes et al., 2013; Hédiji et al., 2015; Lopes Júnior et al., 2014; Sebastian y Prasad, 2015; Wu et al., 2003) frecuentemente han mostrado un efecto depresivo, aunque no siempre significativo, ante la aplicación de $\mathrm{Cd}$.

La influencia de la adición de Cd al sustrato sobre el contenido de $\mathrm{K}$ en las plantas de alfalfa se resume en la Tabla 3. La concentración de $\mathrm{K}$ en la materia seca de raíces aumentó con la dosis de Cd aplicado. Sin embargo, este incremento solo resultó estadísticamente significativo al incrementar la dosis de 5 a $25 \mathrm{mg} \mathrm{Cd} \mathrm{kg} \mathrm{m}^{-1}$ arena, siendo la magnitud del aumento igual a $47 \%$ respecto al testigo. Una tendencia opuesta ha sido reportada para alfalfa (Dražić et al., 2006) y otras especies vegetales (Ahmad et al., 2016; Arshad et al., 2016; Gomes et al., 2013; Hédiji et al., 2015; Li et al., 2016). Sin embargo, algunos 
trabajos (Chaoui et al., 1997; Pérez-Romero et al., 2016) obtuvieron patrones diferentes a esta mayoría. La adición de Cd también provocó un aumento significativo, de hasta $72 \%$ respecto al testigo, en la concentración de $\mathrm{K}$ en la materia seca de la parte aérea. En otros estudios con alfalfa, se han observado resultados similares (Dražić et al., 2006; Ibekwe et al., 1996) o diferentes (Ghnaya et al., 2015). Trabajos realizados con especies vegetales distintas (Ahmad et al., 2016; Arshad et al., 2016; Chaoui et al., 1997; Gomes et al., 2013; Hédiji et al., 2015; Li et al., 2016; Pérez-Romero et al., 2016) han mostrado tendencias variadas.

La variación del contenido de $\mathrm{Mg}$ en las plantas de alfalfa ante la adición de $\mathrm{Cd}$ al sustrato se ilustra en la Tabla 3. La concentración de Mg en la materia seca de raíces tendió a aumentar con la cantidad de $\mathrm{Cd}$ aplicada. El incremento fue de 25 a $31 \%$ respecto al testigo, siendo estadísticamente significativo solamente entre las dosis de 5 a $25 \mathrm{mg} \mathrm{Cd} \mathrm{kg}^{-1}$ arena. La concentración de $\mathrm{Mg}$ en la materia seca de la parte aérea también aumentó progresivay significativamente, hasta en $44 \%$ respecto al testigo, al incrementar el nivel de $\mathrm{Cd}$ en el sustrato. En otros estudios con alfalfa, tendencias opuestas han sido reportadas en raíces (Dražić et al., 2006) y parte aérea (Ghnaya et al., 2015). Sin embargo, un patrón similar al aquí observado también ha sido documentado para la parte aérea (Dražić et al., 2006; Ibekwe et al. 1996). Trabajos con otras plantas (Ahmad et al., 2016; Arshad et al., 2016; Chaoui et al.,
Chaoui et al., 1997; Dias et al., 2013; Gomes et al., 2013; Sebastian y Prasad, 2015; Wu et al., 2003), aunque también tendencias diferentes (Arshad et al., 2016; Lopes Júnior et al., 2014). Por otra parte, al incrementar la dosis de $\mathrm{Cd}$ en la arena, la concentración de $\mathrm{Mn}$ en la materia seca de la parte aérea de las plantas de alfalfa aumentó de manera significativa, hasta en un $66 \%$ respecto al testigo. En alfalfa, Ibekwe et al. (1996) han obtenido resultados similares, pero Motesharezadeh et al. (2010) observaron el patrón opuesto. Una tendencia opuesta ha sido reportada comúnmente en estudios con otras plantas (Ahmad et al., 2016; Arshad et al., 2016; Astolfi et al., 2014; Gomes et al., 2013; Lopes Júnior et al., 2014; Wu et al., 2003), pero existen excepciones (Chaoui et al., 1997; Dias et al., 2013; Sebastian y Prasad, 2015).

La influencia de la aplicación de $\mathrm{Cd}$ al sustrato sobre el contenido de $\mathrm{N}$ en las plantas de alfalfa se muestra en la Tabla 4. La concentración de $\mathrm{N}$ en la materia seca de raíces no fue afectada significativamente por la dosis de Cd aplicado. Sin embargo, la adición de Cd incrementó, hasta en $27 \%$ respecto al testigo, la concentración de $\mathrm{N}$ en la materia seca de la parte aérea, lo cual alcanzó significación estadística. En otros estudios con alfalfa, solo se han reportado efectos no significativos o depresivos del Cd sobre el nivel de $\mathrm{N}$ en la parte aérea (Ghnaya et al., 2015; Ibekwe et al., 1996). En otras plantas, también se han observado las citadas tendencias, tanto en la parte

Tabla 2. Análisis de variancias y promedios para parámetros de contenido de $\mathrm{Ca}, \mathrm{Cu}$ y $\mathrm{Fe}$ en plantas de alfalfa

\begin{tabular}{|c|c|c|c|c|c|c|c|}
\hline \multirow{2}{*}{ Parámetro } & \multicolumn{3}{|c|}{ Cuadrados Medios } & \multirow{2}{*}{$\begin{array}{c}\text { C. V. }{ }^{1} \\
(\%)\end{array}$} & \multicolumn{3}{|c|}{ Promedios para Dosis $\mathrm{Cd}$} \\
\hline & Bloques & Dosis Cd & Error Exp. & & $0 \mathrm{mg} \mathrm{kg}^{-1}$ & $5 \mathrm{mg} \mathrm{kg}^{-1}$ & $25 \mathrm{mg} \mathrm{kg}^{-1}$ \\
\hline Concentración Ca Materia Seca Raíces $\left(\mathrm{g} \mathrm{kg}^{-1}\right)$ & $0,0297^{\mathrm{NS}, 2}$ & $2,3815^{\mathrm{NS}}$ & 0,7656 & 16,27 & $5,24 \mathrm{a}^{3}$ & $4,56 a$ & $6,33 a$ \\
\hline Concentración Ca Materia Seca Parte Aérea $\left(\mathrm{g} \mathrm{kg}^{-1}\right)$ & 8,01 NS & $39,71 *$ & 1,93 & 5,46 & $21,3 b$ & $26,8 \mathrm{a}$ & $28,2 \mathrm{a}$ \\
\hline Extracción Ca Total (mg maceta $\left.{ }^{-1}\right)$ & $767^{\mathrm{NS}}$ & $291 \mathrm{NS}$ & 484 & 15,03 & $149 \mathrm{a}$ & $155 \mathrm{a}$ & $135 \mathrm{a}$ \\
\hline Extracción Ca Parte Aérea/Raíces & 0,765 NS & $12,333^{*}$ & 1,427 & 12,35 & $7,61 b$ & $11,67 \mathrm{a}$ & $9,73 \mathrm{ab}$ \\
\hline Concentración $\mathrm{Cu}$ Materia Seca Raíces $\left(\mathrm{mg} \mathrm{kg}^{-1}\right)$ & 2,73 NS & 161,95 NS & 41,15 & 20,84 & $24,6 a$ & $28,9 \mathrm{a}$ & $38,9 \mathrm{a}$ \\
\hline Concentración $\mathrm{Cu}$ Materia Seca Parte Aérea $\left(\mathrm{mg} \mathrm{kg}^{-1}\right)$ & $0,930^{\mathrm{NS}}$ & $0,816^{\mathrm{NS}}$ & 0,422 & 8,10 & $8,61 \mathrm{a}$ & $7,82 \mathrm{a}$ & $7,63 \mathrm{a}$ \\
\hline Extracción $\mathrm{Cu}$ Total $\left(\mu \mathrm{g}\right.$ maceta $\left.^{-1}\right)$ & 159,2 NS & 377,1 Ns & 97,4 & 8,18 & $133 \mathrm{a}$ & $118 \mathrm{a}$ & $111 \mathrm{a}$ \\
\hline Extracción Cu Parte Aérea/Raíces & 0,00373 NS & $0,03645^{\mathrm{NS}}$ & 0,00526 & 13,37 & $0,650 \mathrm{a}$ & $0,547 \mathrm{ab}$ & $0,430 \mathrm{~b}$ \\
\hline Concentración Fe Materia Seca Parte Aérea $\left(\mathrm{mg} \mathrm{kg}^{-1}\right)$ & $13,8 \mathrm{NS}$ & $77,5^{\mathrm{NS}}$ & 108,1 & 15,79 & $60,4 a$ & $70,5 \mathrm{a}$ & $66,6 a$ \\
\hline Extracción Fe Parte Aérea ( $\mu$ g maceta $\left.{ }^{-1}\right)$ & $4192^{\text {NS }}$ & 6838 NS & 6953 & 24,06 & $376 a$ & $373 a$ & 291a \\
\hline
\end{tabular}

1 Coeficiente de variabilidad.

2 Prueba de F para $\alpha$ igual a 0,05 : no significativa (NS) o significativa $(*)$.

3 Prueba de Tukey entre promedios para $\alpha$ igual a 0,05: letras distintas indican grupos de tratamientos significativamente diferentes.

1997; Dias et al., 2013; Gomes et al., 2013; Hédiji et al., 2015; Li et al., 2016; Lopes Júnior et al., 2014; PérezRomero et al., 2016) han producido resultados variados, tanto para raíces como parte aérea.

El efecto de la aplicación de Cd sobre el contenido de Mn en las plantas de alfalfa se presenta en la Tabla 3. La concentración de $\mathrm{Mn}$ en la materia seca de raíces sufrió un leve y estadísticamente intrascendente aumento ante la aplicación de Cd. Motesharezadeh et al. (2010) tampoco observaron efecto significativo alguno en alfalfa. En otras especies vegetales la adición de $\mathrm{Cd}$ al medio frecuentemente ha provocado una disminución de la concentración de Mn en las raíces (Ahmad et al., 2016; Astolfi et al., 2014; aérea como en raíces (Gill et al., 2012; Gomes et al., 2013; Li et al., 2016). Debe tenerse presente que todas las plantas de este estudio presentaron nódulos fijadores de $\mathrm{N}_{2}$ en las raíces. Nodulación en raíces de alfalfa desarrollando en suelos altamente contaminados también ha sido documentada por Angle y Chaney (1991).

La variación del contenido de $\mathrm{Na}$ en las plantas de alfalfa ante la adición de $\mathrm{Cd}$ al sustrato se presenta en la Tabla 4. La concentración de $\mathrm{Na}$ en la materia seca de raíces sufrió una reducción marcada, hasta un $68 \%$ del valor del testigo, al aplicar $\mathrm{Cd}$ al medio. Esta disminución no logró significación con las pruebas estadísticas usadas. Por otra parte, el incremento del nivel de $\mathrm{Cd}$ en el sustrato 
provocó un aumento de $57 \%$, respecto al testigo, en la concentración de $\mathrm{Na}$ en la materia seca de la parte aérea. Esta diferencia tampoco alcanzó significación con las pruebas estadísticas empleadas. No se pudieron encontrar reportes relacionados para alfalfa en la literatura. En otras plantas, se ha observado que la adición de Cd provocó una reducción de la concentración de $\mathrm{Na}$ en raíces (Li et al., 2016; Pérez-Romero et al., 2016) y ninguna tendencia clara en la parte aérea (Pérez-Romero et al., 2016).

El efecto de la adición de Cd sobre el contenido de $\mathrm{P}$ en las plantas de alfalfa se ilustra en la Tabla 4. La concentración de $\mathrm{P}$ en la materia seca de raíces no fue afectada significativamente por la dosis de Cd. En cambio, la aplicación de $\mathrm{Cd}$ incrementó, en hasta $30 \%$ respecto al testigo, la concentración de $\mathrm{P}$ en la materia seca de la parte aérea. Esta diferencia alcanzó significación estadística. Ibekwe et al. (1996) observó la misma tendencia para la parte aérea de plantas de alfalfa. Estudios con otras plantas (Arshad et al., 2016; Gomes et al., 2013; Li et al., 2016; Lopes Júnior et al., 2014) han mostrado efectos variados, tanto en parte aérea como raíces.

La influencia de la aplicación de $\mathrm{Cd}$ al sustrato sobre el contenido de $\mathrm{S}$ en las plantas de alfalfa se presenta en la Tabla 5. La concentración de $\mathrm{S}$ en la materia seca de raíces aumentó en un $57 \%$, respecto al testigo, al variar la dosis de adición de Cd de 5 a $25 \mathrm{mg} \mathrm{kg}^{-1}$. Este incremento no alcanzó significación con las pruebas estadísticas usadas. Además, la adición de $\mathrm{Cd}$ incrementó de manera significativa, en hasta $42 \%$ respecto al testigo, la concentración de $\mathrm{S}$ en la materia seca de la parte aérea. Reportes relacionados no se han podido encontrar en la literatura de la alfalfa. En estudios con otras plantas (Ahmad et al., 2016; Gill et al., 2012; Gomes et al., 2013) se han observado efectos variados sobre la concentración de $\mathrm{S}$ en tejidos vegetales ante la adición de $\mathrm{Cd}$.

La variación del contenido de $\mathrm{Zn}$ en las plantas de alfalfa ante la adición de $\mathrm{Cd}$ al sustrato se presenta en la Tabla 5. Las concentraciones de $\mathrm{Zn}$ en la materia seca de raíces y parte aérea no fueron afectadas significativamente por la dosis de Cd aplicada. Motesharezadeh et al. (2010) reportó un aumento de la concentración de $\mathrm{Zn}$ en el tejido radicular de alfalfa al suministrar $\mathrm{Cd}$, pero ningún efecto significativo de este sobre el nivel de Zn en la parte aérea. Reportes en otras especies de plantas (Astolfi et al., 2014; Chaoui et al., 1997; Gomes et al., 2013; Hédiji et al., 2015; Wu et al., 2003) no han mostrado ningún patrón generalizable.

Las cantidades de nutrientes minerales absorbidas por las plantas de alfalfa se presentan en las Tablas 2 a 5 . Al enriquecer el sustrato con $\mathrm{Cd}$, las extracciones totales de $\mathrm{Ca}, \mathrm{Cu}, \mathrm{Mg}, \mathrm{Mn}, \mathrm{N}, \mathrm{Na}, \mathrm{P}$ y Zn y la extracción de la parte aérea de $\mathrm{Fe}$ tendieron a disminuir, siendo estadísticamente significativos solo para $\mathrm{Mn}, \mathrm{Na}$ y $\mathrm{Zn}$. En cambio, K y $\mathrm{S}$ mostraron patrones diferentes y estadísticamente no importantes. Plantas expuestas al Cd tuvieron la oportunidad de extraer cantidades similares de elementos nutritivos que individuos testigo y tendieron a concentrarlas en su reducida materia seca. Por otra parte, al adicionar $\mathrm{Cd}$ al sustrato, los cocientes de extracción de parte aérea a raíces de $\mathrm{Ca}, \mathrm{K}, \mathrm{Mg}, \mathrm{Mn}, \mathrm{N}, \mathrm{Na}, \mathrm{P}$ y Zn de las plantas de alfalfa tendieron a elevarse, logrando importancia estadística solo para $\mathrm{Ca}$ y $\mathrm{Mg}$. En cambio, los valores respectivos para $\mathrm{Cu}$ disminuyeron significativamente, mientras que aquellos para el $\mathrm{S}$ no mostraron una tendencia simple ni estadísticamente relevante. Esto indica que las plantas de alfalfa tendieron a movilizar la mayoría de los elementos nutritivos a la parte aérea a expensas de las raíces.

El estado nutricional general de las plantas de alfalfa de este experimento se evaluó mediante comparación de las concentraciones de elementos nutritivos en la parte aérea de individuos no tratados con $\mathrm{Cd}$ con los datos compilados por Pinkerton et al. (1997) para plantas similares. Esta evaluación sugiere que el crecimiento de individuos testigo no fue limitado por el suministro de $\mathrm{Ca}, \mathrm{Cu}, \mathrm{Fe}, \mathrm{K}, \mathrm{Mg}$, $\mathrm{Mn}, \mathrm{N}, \mathrm{P}, \mathrm{S}$ o $\mathrm{Zn}$ del sustrato. Lo mismo es válido para plantas sujetas a una dosis de $25 \mathrm{mg} \mathrm{Cd} \mathrm{kg}^{-1}$ arena. Por lo tanto, ninguna de las plantas de alfalfa de este estudio parece haber sufrido deficiencia o toxicidad de alguno de los elementos nutritivos estudiados.

Un mecanismo común de interacción entre el $\mathrm{Cd}$ y otros elementos químicos en las plantas es la competencia al ser absorbidos por las raíces (DalCorso et al., 2013; Irfan et al., 2013). Considerando que la extracción total máxima de $\mathrm{Cd}$ en este trabajo fue $0,0133 \mathrm{mmol} \mathrm{maceta}^{-1}$, solo $\mathrm{Cu}, \mathrm{Mn}, \mathrm{Zn}$ y, eventualmente, $\mathrm{Fe}$ podrían haber sido afectados. Extracciones totales de otros elementos nutritivos potencialmente competitivos superaron el valor indicado para el Cd en mas de 100 veces. Las extracciones totales de $\mathrm{Cu}, \mathrm{Mn}$ y $\mathrm{Zn}$ disminuyeron al aumentar la dosis de $\mathrm{Cd}$ en el sustrato, alcanzando significación estadística solo para Mn y Zn. Sin embargo, los niveles de Mn y Zn en la parte aérea de las plantas expuestas al $\mathrm{Cd}$ fueron superiores que o comparables con aquellos de plantas testigo, respectivamente. Por otra parte, la concentración de $\mathrm{Cu}$ en la parte aérea no calificó como deficiente. Por lo tanto, efectos depresivos específicos del Cd sobre la absorción de $\mathrm{Cu}, \mathrm{Mn}$ y $\mathrm{Zn}$ no parecen haber ocurrido en el presente estudio, excepto quizás para el $\mathrm{Cu}$.

La ocurrencia de efectos específicos del $\mathrm{Cd}$ sobre nutrientes minerales en plantas parece depender de las condiciones particulares del estudio. Esto explicaría la diversidad de respuestas reportadas en la literatura, tanto en alfalfa como otras especies vegetales. Además, se ha observado que el estrés por toxicidad de $\mathrm{Cd}$ puede ser reducido mediante la adición de Ca en garbanzo (Ahmad et al., 2016), Fe en arroz (Sebastian y Prasad, 2015), K en garbanzo (Ahmad et al., 2016), Mn en arroz (Sebastian y Prasad, 2015), $\mathrm{N}$ en álamo (Zhang et al., 2014), $\mathrm{P}$ en arroz (Yang et al., 2016) y S en mostaza (Bashir et al., 2015). El éxito individual de alternativas tan diversas para aliviar los síntomas del mismo problema sugiere la falta de especificidad en la interacción de $\mathrm{Cd}$ con elementos nutritivos de plantas. Es posible que dosis de aplicación mas altas que $25 \mathrm{mg} \mathrm{Cd} \mathrm{kg}^{-1}$ arena sean requeridas para que las plantas de alfalfa muestren de manera marcada efectos específicos del Cd sobre algún nutriente mineral. 
Tabla 3. Análisis de variancias y promedios para parámetros de contenido de $\mathrm{K}, \mathrm{Mg}$ y Mn en plantas de alfalfa

\begin{tabular}{|c|c|c|c|c|c|c|c|}
\hline \multirow{2}{*}{ Parámetro } & \multicolumn{3}{|c|}{ Cuadrados Medios } & \multirow{2}{*}{$\begin{array}{c}\text { C. V. }{ }^{1} \\
(\%)\end{array}$} & \multicolumn{3}{|c|}{ Promedios para Dosis Cd } \\
\hline & Bloques & Dosis Cd & Error Exp. & & $0 \mathrm{mg} \mathrm{kg}^{-1}$ & $5 \mathrm{mg} \mathrm{kg}^{-1}$ & $25 \mathrm{mg} \mathrm{kg}^{-1}$ \\
\hline Concentración K Materia Seca Raíces $\left(\mathrm{g} \mathrm{kg}^{-1}\right)$ & $2,06^{\mathrm{NS}, 2}$ & $23,60^{*}$ & 1,58 & 8,77 & $13,8 a b^{3}$ & $11,8 b$ & $17,4 \mathrm{a}$ \\
\hline Concentración K Materia Seca Parte Aérea $\left(\mathrm{g} \mathrm{kg}^{-1}\right)$ & $6,66^{\mathrm{NS}}$ & $73,71^{*}$ & 2,50 & 8,87 & $13,5 b$ & $16,7 b$ & $23,2 \mathrm{a}$ \\
\hline Extracción K Total (mg maceta ${ }^{-1}$ ) & $178^{\mathrm{NS}}$ & $131^{\mathrm{NS}}$ & 271 & 12,82 & $128 \mathrm{a}$ & $122 \mathrm{a}$ & $135 \mathrm{a}$ \\
\hline Extracción K Parte Aérea/Raíces & 0,497 NS & $1,205^{*}$ & 0,162 & 15,87 & $1,80 \mathrm{a}$ & $2,91 \mathrm{a}$ & $2,90 \mathrm{a}$ \\
\hline Concentración Mg Materia Seca Raíces $\left(\mathrm{g} \mathrm{kg}^{-1}\right)$ & $0,168^{\mathrm{NS}}$ & $3,806^{*}$ & 0,352 & 7,81 & $7,12 b$ & $6,79 b$ & $8,89 \mathrm{a}$ \\
\hline Concentración Mg Materia Seca Parte Aérea $\left(\mathrm{g} \mathrm{kg}^{-1}\right)$ & $0,0243^{\mathrm{NS}}$ & $1,4145^{*}$ & 0,0315 & 4,66 & $3,07 \mathrm{~b}$ & $3,93 \mathrm{a}$ & $4,43 \mathrm{a}$ \\
\hline Extracción Mg Total (mg maceta $\left.{ }^{-1}\right)$ & $59,6^{\mathrm{NS}}$ & 23,2 Ns & 27,2 & 13,13 & $42,6 \mathrm{a}$ & $39,5 \mathrm{a}$ & $37,1 \mathrm{a}$ \\
\hline Extracción Mg Parte Aérea/Raíces & $0,03268 \mathrm{NS}$ & $0,11191 *$ & 0,00878 & 9,26 & $0,793 b$ & $1,158 \mathrm{a}$ & $1,086 \mathrm{a}$ \\
\hline Concentración Mn Materia Seca Raíces (mg kg-1) & $557^{\mathrm{NS}}$ & $3104^{\mathrm{NS}}$ & 14023 & 28,20 & $385 \mathrm{a}$ & $448 \mathrm{a}$ & $428 \mathrm{a}$ \\
\hline Concentración Mn Materia Seca Parte Aérea $\left(\mathrm{mg} \mathrm{kg}^{-1}\right)$ & $17,2 \mathrm{NS}$ & $2600,1 *$ & 36,1 & 5,11 & $89,2 \mathrm{c}$ & $115,7 b$ & $148,0 \mathrm{a}$ \\
\hline Extracción Mn Total $\left(\mu \mathrm{g}\right.$ maceta $\left.^{-1}\right)$ & $50569^{*}$ & $100279^{*}$ & 3787 & 3,63 & $1810 \mathrm{a}$ & $1792 \mathrm{a}$ & $1485 b$ \\
\hline Extracción Mn Parte Aérea/Raíces & $0,0150^{\mathrm{NS}}$ & $0,1080^{\mathrm{NS}}$ & 0,0195 & 24,06 & $0,428 \mathrm{a}$ & $0,522 \mathrm{a}$ & $0,793 \mathrm{a}$ \\
\hline
\end{tabular}

1 Coeficiente de variabilidad.

2 Prueba de F para $\alpha$ igual a 0,05 : no significativa (NS) o significativa $(*)$.

3 Prueba de Tukey entre promedios para $\alpha$ igual a 0,05: letras distintas indican grupos de tratamientos significativamente diferentes.

Tabla 4. Análisis de variancias y promedios para parámetros de contenido de N, Na y P en plantas de alfalfa

\begin{tabular}{|c|c|c|c|c|c|c|c|}
\hline \multirow{2}{*}{ Parámetro } & \multicolumn{3}{|c|}{ Cuadrados Medios } & \multirow{2}{*}{$\begin{array}{c}\text { C. V. }{ }^{1} \\
(\%)\end{array}$} & \multicolumn{3}{|c|}{ Promedios para Dosis Cd } \\
\hline & Bloques & Dosis Cd & Error Exp. & & $0 \mathrm{mg} \mathrm{kg}^{-1}$ & $5 \mathrm{mg} \mathrm{kg}^{-1}$ & $25 \mathrm{mg} \mathrm{kg}^{-1}$ \\
\hline Concentración N Materia Seca Raíces $\left(\mathrm{g} \mathrm{kg}^{-1}\right)$ & $10,17^{\mathrm{NS}, 2}$ & 3,41 NS & 3,37 & 7,05 & $25,9 \mathrm{a}^{3}$ & $27,1 \mathrm{a}$ & $25,0 \mathrm{a}$ \\
\hline Concentración N Materia Seca Parte Aérea $\left(\mathrm{g} \mathrm{kg}^{-1}\right)$ & 7,05 Ns & $55,95^{*}$ & 5,73 & 6,44 & $32,7 b$ & $41,4 \mathrm{a}$ & $37,4 a$ \\
\hline Extracción N Total (mg maceta $\left.{ }^{-1}\right)$ & $3669^{\mathrm{NS}}$ & $6117^{\mathrm{NS}}$ & 1590 & 14,99 & $288 \mathrm{a}$ & $295 \mathrm{a}$ & $214 \mathrm{a}$ \\
\hline Extracción N Parte Aérea/Raíces & $0,263 \mathrm{NS}$ & 0,763 NS & 0,272 & 18,01 & $2,32 \mathrm{a}$ & $3,11 \mathrm{a}$ & $3,25 \mathrm{a}$ \\
\hline Concentración Na Materia Seca Raíces $\left(\mathrm{g} \mathrm{kg}^{-1}\right)$ & 1,61 Ns & 7,55 Ns & 2,78 & 22,28 & $9,29 \mathrm{a}$ & $6,29 \mathrm{a}$ & $6,88 \mathrm{a}$ \\
\hline Concentración Na Materia Seca Parte Aérea $\left(\mathrm{g} \mathrm{kg}^{-1}\right)$ & $0,285^{\mathrm{NS}}$ & $0,572 \mathrm{NS}$ & 0,219 & 31,42 & $1,27 \mathrm{a}$ & $1,21 \mathrm{a}$ & $1,99 \mathrm{a}$ \\
\hline Extracción Na Total (mg maceta $\left.{ }^{-1}\right)$ & $128,1^{\mathrm{NS}}$ & $276,9^{*}$ & 33,7 & 20,56 & $39,3 \mathrm{a}$ & $23,1 \mathrm{ab}$ & $22,3 b$ \\
\hline Extracción Na Parte Aérea/Raíces & 0,0153 NS & $0,1096^{\mathrm{NS}}$ & 0,0254 & 37,56 & $0,256 \mathrm{a}$ & $0,386 \mathrm{a}$ & $0,632 \mathrm{a}$ \\
\hline Concentración P Materia Seca Raíces $\left(\mathrm{g} \mathrm{kg}^{-1}\right)$ & $0,0289^{\mathrm{NS}}$ & $0,0495^{\mathrm{NS}}$ & 0,0689 & 9,44 & $2,64 \mathrm{a}$ & $2,81 \mathrm{a}$ & $2,89 \mathrm{a}$ \\
\hline Concentración P Materia Seca Parte Aérea $\left(\mathrm{g} \mathrm{kg}^{-1}\right)$ & 0,1970 Ns & $0,3435^{*}$ & 0,0349 & 7,23 & $2,23 b$ & $2,64 \mathrm{ab}$ & $2,90 \mathrm{a}$ \\
\hline Extracción P Total (mg maceta $\left.{ }^{-1}\right)$ & 1,23 NS & $12,47^{\mathrm{NS}}$ & 4,98 & 10,77 & $22,2 \mathrm{a}$ & $21,6 \mathrm{a}$ & $18,4 \mathrm{a}$ \\
\hline Extracción P Parte Aérea/Raíces & $0,0977^{\mathrm{NS}}$ & $0,3088^{\mathrm{NS}}$ & 0,0813 & 15,22 & $1,54 \mathrm{a}$ & $1,90 \mathrm{a}$ & $2,18 \mathrm{a}$ \\
\hline
\end{tabular}

Extracción P Parte Aérea/Raíces

1 Coeficiente de variabilidad.

2 Prueba de F para $\alpha$ igual a 0,05 : no significativa (NS) o significativa $(*)$.

3 Pueba de Tukey entre promedios para $\alpha$ igual a 0,05: letras distintas indican grupos de tratamientos significativamente diferentes.

Tabla 5. Análisis de variancias y promedios para parámetros de contenido de S y Zn en plantas de alfalfa

\begin{tabular}{|c|c|c|c|c|c|c|c|}
\hline \multirow{2}{*}{ Parámetro } & \multicolumn{3}{|c|}{ Cuadrados Medios } & \multirow{2}{*}{$\begin{array}{l}\text { C. V. }{ }^{1} \\
(\%)\end{array}$} & \multicolumn{3}{|c|}{ Promedios para Dosis $\mathrm{Cd}$} \\
\hline & Bloques & Dosis Cd & Error Exp. & & $0 \mathrm{mg} \mathrm{kg}^{-1}$ & $5 \mathrm{mg} \mathrm{kg}^{-1}$ & $25 \mathrm{mg} \mathrm{kg}^{-1}$ \\
\hline Concentración S Materia Seca Raíces $\left(\mathrm{g} \mathrm{kg}^{-1}\right)$ & $2,46^{\mathrm{NS}, 2}$ & 8,33 NS & 1,22 & 17,23 & $5,58 \mathrm{a}^{3}$ & $5,32 \mathrm{a}$ & $8,33 \mathrm{a}$ \\
\hline Concentración S Materia Seca Parte Aérea $\left(\mathrm{g} \mathrm{kg}^{-1}\right)$ & $0,136^{\mathrm{NS}}$ & $1,762 \mathrm{NS}$ & 0,268 & 11,57 & $3,70 b$ & $4,47 \mathrm{ab}$ & $5,24 \mathrm{a}$ \\
\hline Extracción S Total (mg maceta $\left.{ }^{-1}\right)$ & $11,11^{\mathrm{NS}}$ & $9,16^{\mathrm{NS}}$ & 24,45 & 12,53 & $41,3 \mathrm{a}$ & $37,8 \mathrm{a}$ & $39,4 \mathrm{a}$ \\
\hline Extracción S Parte Aérea/Raíces & $0,1498^{\mathrm{NS}}$ & 0,1709 NS & 0,0649 & 17,47 & $1,28 \mathrm{a}$ & $1,73 \mathrm{a}$ & $1,37 \mathrm{a}$ \\
\hline Concentración Zn Materia Seca Raíces ( $\left.\mathrm{mg} \mathrm{kg}^{-1}\right)$ & $33,8^{\mathrm{NS}}$ & $15,4^{\mathrm{NS}}$ & 39,2 & 13,22 & $44,9 \mathrm{a}$ & $48,0 \mathrm{a}$ & $49,3 \mathrm{a}$ \\
\hline Concentración Zn Materia Seca Parte Aérea $\left(\mathrm{mg} \mathrm{kg}^{-1}\right)$ & $0,803^{\mathrm{NS}}$ & $2,596^{\mathrm{NS}}$ & 1,973 & 7,20 & $19,5 \mathrm{a}$ & $18,5 \mathrm{a}$ & $20,4 \mathrm{a}$ \\
\hline Extracción Zn Total ( $\mu$ g maceta $\left.^{-1}\right)$ & $645^{\mathrm{NS}}$ & $4656^{*}$ & 154 & 5,47 & $266 \mathrm{a}$ & $226 b$ & $188 \mathrm{c}$ \\
\hline Extracción Zn Parte Aérea/Raíces & $0,005644^{\mathrm{NS}}$ & $0,01240^{\mathrm{NS}}$ & 0,00769 & 10,59 & $0,807 \mathrm{a}$ & $0,777 \mathrm{a}$ & $0,901 \mathrm{a}$ \\
\hline
\end{tabular}

1 Coeficiente de variabilidad.

2 Prueba de F para $\alpha$ igual a 0,05 : no significativa (NS) o significativa $(*)$.

3 Prueba de Tukey entre promedios para $\alpha$ igual a 0,05 : letras distintas indican grupos de tratamientos significativamente diferentes.

\section{Conclusiones}

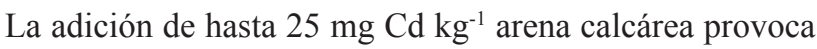
una notoria disminución en el crecimiento de plantas de alfalfa, pero sin lograr un efecto tóxico grave. Ningún efecto específico del $\mathrm{Cd}$ aplicado fue notado sobre la nutrición de $\mathrm{Ca}, \mathrm{Fe}, \mathrm{K}, \mathrm{Mg}, \mathrm{Mn}, \mathrm{K}, \mathrm{N}, \mathrm{Na}, \mathrm{P}, \mathrm{S}$ y Zn en las plantas. Sin embargo, un leve efecto depresivo pudo haber ocurrido con el $\mathrm{Cu}$. La adición de Cd tuvo un efecto casi generalizado de concentración de estos elementos en la materia seca de raíces y parte aérea. Además, las plantas tendieron a movilizar los nutrientes minerales hacia la parte 
aérea a expensas de las raíces. Aparentemente, el efecto principal del $\mathrm{Cd}$ fue reducir directamente la fotosíntesis. Posiblemente, una intoxicación mas grave con $\mathrm{Cd}$ logre provocar interacciones mas claras de este elemento con nutrientes minerales.

\section{Agradecimientos}

El autor agradece al Laboratorio de Análisis de Suelos, Plantas, Aguas y Fertilizantes de la Facultad de Agronomía de la Universidad Nacional Agraria La Molina por darle las facilidades necesarias para realizar ciertos análisis y ayudar con determinaciones analíticas en el sustrato usado y en plantas de alfalfa.

\section{Literatura citada}

Ahmad, P.; Abdel, A.A.; Abd_Allah, E.F.; Hashem, A.; Sarwat, M.; Anjum, N.A. and Gucel, S. 2016. Calcium and potassium supplementation enhanced growth, osmolyte secondary metabolite production, and enzymatic antioxidant machinery in cadmium-exposed chickpea (Cicer arietinum L.). Frontiers in Plant Science, 7:513. doi: 10.3389/fpls.2016.00513.

Angle, J.S. and Chaney, R.L. 1991. Heavy metal effects on soil populations and heavy metal tolerance of Rhizobium meliloti, nodulation, and growth of alfalfa. Water, Air, and Soil Pollution, 57 (1):597-604.

Arshad, M.; Ali, S.; Noman, A.; Ali, Q.; Rizwan, M.; Farid, M. and Irshad, M.K. 2016. Phosphorus amendment decreased cadmium (Cd) uptake and ameliorates chlorophyll contents, gas exchange attributes, antioxidants, and mineral nutrients in wheat (Triticum aestivum L.) under Cd stress. Archives of Agronomy and Soil Science, 62 (4):533-546.

Astolfi, S.; Ortolani, M.R.; Catarcione, G.; Paolacci, A.R.; Cesco, S.; Pinton, R. and Ciaffi, M. 2014. Cadmium exposure affects iron acquisition in barley (Hordeum vulgare) seedlings. Physiologia Plantarum, 152 (4):646-659.

Bashir, H.; Ibrahim, M.M.; Bagheri, R.; Ahmad, J.; Arif, I.A.; Baig, M.A. and Qureshi, M.I. 2015. Influence of sulfur and cadmium on antioxidants, phytochelatins, and growth in Indian mustard. AoB Plants, 7:1. doi:10.1093/aobpla/plv001.

Bazán, T. R. 1996. Manual para el análisis químico de suelos, aguas y plantas. Universidad Nacional Agraria La Molina - Fundación Perú, Lima, Perú. 55 p.

Bingham, F.T.; Page, A.L.; Mahler, R.J. and Ganje, T.J. 1975. Growth and cadmium accumulation of plants grown on a soil treated with a cadmium-enriched sewage sludge. Journal of Environmental Quality, 4 (2):207-211.

Borišev, M.; Pajević, S.; Nikolić, N.; Orlović, S.; Župunski, N.; Pilipović, A. and Kerbert, M. 2016. Magnesium and iron deficiencies alter $\mathrm{Cd}$ accumulation in Salix viminalis L. International Journal of Phytoremediation,
18 (2):164-170.

Chaoui, A.; Ghorbal, M.H. and El Ferjani, E. 1997. Effects of cadmium-zinc interactions on hydroponically grown bean (Phaseolus vulgaris L.). Plant Science, 126 (1): 21-28.

DalCorso, G.; Manara, A. and Furini, A. 2013. An overview of heavy metal challenge in plants: From roots to shoots. Metallomics, 5:1117-1132.

Dias, M.C.; Monteiro, C.; Moutinho-Pereira, J.; Correia, C.; Gonçalves, B. and Santos, C. 2013. Cadmium toxicity affects photosynthesis and plant growth at different levels. Acta Physiologiae Plantarum, 35 (4):1281-1289.

Dražić, G.; Mihailović, N. and Lojić, M. 2006. Cadmium accumulation in Medicago sativa seedlings treated with salicylic acid. Biologia Plantarum, 50 (2):239-244.

Eller, F. \& Brix, H. 2016. Influence of low calcium availability on cadmium uptake and translocation in a fast-growing shrub and a metal-accumulating herb. AoB Plants, 8:143. doi:10.1093/aobpla/plv143.

Elouear, Z.; Bouhamed, F.; Boujelben, N. and Bouzid, J. 2016. Application of sheep manure and potassium fertilizer to contaminated soil and its effect on zinc, cadmium and lead accumulation by alfalfa plants. Sustainable Environment Research, 26 (3):131-135.

Ghnaya, T.; Mnassri, M.; Ghabriche, R.; Wali, M.; Poschenrieder, C.; Lutts, S. and Abdelly, C. 2015. Nodulation by Sinorhizobium meliloti originated from a mining soil alleviates $\mathrm{Cd}$ toxicity and increases $\mathrm{Cd}$ phytoextraction in Medicago sativa L. Frontiers in Plant Science, 6:863. doi 10.3389/fpls.2015.00863.

Gill, S.S.; Khan, N.A. and Tuteja, N. 2012. Cadmium at high dose perturbs growth, photosynthesis and nitrogen metabolism while at low dose it up regulates sulfur assimilation and antioxidant machinery in garden cress (Lepidium sativum L.). Plant Science, 182:112-120.

Gomes, M.P.; Marques, T. and Soares, A.M. 2013. Cadmium effects on mineral nutrition of the $\mathrm{Cd}$ hyperaccumulator Pfaffia glomerata. Biologia, 68 (2):223-230.

Hédiji, H.; Djebali, W.; Belkadhi, A.; Cabasson, C.; Moing, A.; Rolin, D.; Brouquisse, R.; Gallusci, P. and Chaibi, W. 2015. Impact of long-term cadmium exposure on mineral content of Solanum lycopersicum plants: Consequences on fruit production. South African Journal of Botany ,97:176-181.

Hollander, M.; Wolfe, D.A. and Chicken, E. 2014. Nonparametric statistical methods. 3ra Edición. John Wiley \& Sons, Hoboken, New Jersey, EEUU. 819 p.

Ibekwe, A.M.; Angle, J.S.; Chaney, R.L. and Van Berkum, P. 1996. Zinc and cadmium toxicity to alfalfa and its microsymbiont. Journal of Environmental Quality, 25 (5):1032-1040.

Irfan, M.; Hayat, S.; Ahmad, A. and Alyemeni, M.N. 2013. Soil cadmium enrichment: Allocations and 
plant physiological manifestations. Saudi Journal of Biological Sciences, 20 (1):1-10.

Jones, J.B. and Case, V.W. 1990. Sampling, handling, and analyzing plant tissue samples. En: Westerman (Comp.). Soil testing and plant analysis. 3ra Edición. Soil Science Society of America Book Series No. 3. Soil Science Society of America, Madison, Wisconsin, EEUU. 389-427p.

Kabata-Pendias, A. and Szteke, B. 2015. Trace elements in abiotic and biotic environments. CRC Press, Boca Raton, Florida, EEUU. 505 pp.

Li, P.; Zhao, C.; Zhang, Y.; Wang, X.; Wang, X.; Wang, J.; Wang, F. and Bi, Y. 2016. Calcium alleviates cadmiuminduced inhibition on root growth by maintaining auxin homeostasis in Arabidopsis seedlings. Protoplasma, 253(1):185-200.

Lopes Júnior, C.A.; Mazzafera, P. and Zezzi, M.A. 2014. A comparative ionomic approach focusing on cadmium effects in sunflowers (Helianthus annuus L.). Environmental and Experimental Botany, 107:180-186.

Motesharezadeh, B.; Savaghebi-Firoozabadi, G.R.; Mirseyed Hosseini, H. and Alikhani, H.A. 2010. Study of the enhanced phytoextraction of cadmium in a calcareous soil. International Journal of Environmental Research, 4(3):525-532.

Ott, R.L. and Longnecker, M.T. 2016. An introduction to statistical methods and data analysis. 7ma Edición. Cengage Learning, Boston, Massachusetts, EEUU. 1174 p.

Pérez-Romero, J.A.; Redondo-Gómez, S. and MateosNaranjo, E. 2016. Growth and photosynthetic limitation analysis of the Cd-accumulator Salicornia ramosissima under excessive cadmium concentrations and optimum salinity conditions. Plant Physiology and Biochemistry, 109:103-113.

Pinkerton, A.; Smith, F.W. and Lewis, D.C. 1997. 6. Pasture species. En: Reuter, D.J.; Robinson, J.B. (Comp.). Plant analysis: An interpretation manual. 2da. Edición. CSIRO Publishing, Collingwood, Victoria, Australia. pp. 287-346.

Sebastian, A. and Prasad, M.N.V. 2015. Iron- and manganese-assisted cadmium tolerance in Oryza sativa L.: Lowering the rhizotoxicity next to functional photosynthesis. Planta, 241 (6):1519-1528.

Wu, F.; Zhang, G. and Yu, J. 2003. Interaction of cadmium and four microelements for uptake and translocation in different barley genotypes. Communications in Soil Science and Plant Analysis, 34 (13):2003-2020.

Yang, Y.; Chen, R.; Fu, G.; Xiong, J. and Tao, L. 2016. Phosphate deprivation decreases cadmium $(\mathrm{Cd})$ uptake but enhances sensitivity to $\mathrm{Cd}$ by increasing iron $(\mathrm{Fe})$ uptake and inhibiting phytochelatins synthesis in rice (Oryza sativa). Acta Physiologiae Plantarum, 38:28. doi:10.1007/s11738-015-2055-9.

Zhang, F.; Wan, X.; Zheng, Y.; Sun, L.; Chen, Q.; Zhu, X.;
Guo, Y. and Liu, M. 2014. Effects of nitrogen on the activity of antioxidant enzymes and gene expression in leaves of Populus plants subjected to cadmium stress. Journal of Plant Interactions, 9 (1):599-609. 